\title{
Correction to: Diaspora and Ethnic Contestation in Guyana
}

Ralph Premdas and Bishnu Ragoonath

\section{Correction to: Chapter 69 in: S. Ratuva (ed.), The Palgrave Handbook of Ethnicity, https://doi.org/10.1007/978-981-13-2898-5_45}

The co-author name has been inadvertently retained as "Ragoonat" and the same has been updated now as "Ragoonath" in the chapter. 